\title{
UNIQUENESS OF CERTAIN TYPE OF DIFFERENTIAL-DIFFERENCE AND DIFFERENCE POLYNOMIALS
}

\author{
ABHIJIT BANERJEE AND SUJOY MAJUMDER
}

\begin{abstract}
In this paper we consider certain difference and differential-difference polynomials sharing some polynomial and improve a number of results in [9], [11] and [17]. In particular we point out a gap in the argument in the proof of the main results in [11] and rectifying the same we improve and extend the result to a large extent.
\end{abstract}

\section{Introduction, Definitions and Results}

In this paper by meromorphic functions we shall always mean meromorphic functions in the complex plane.

We adopt the standard notations of value distribution theory (see [4]). For a non-constant meromorphic function $f$, we denote by $T(r, f)$ the Nevanlinna characteristic of $f$ and by $S(r, f)$ any quantity satisfying $S(r, f)=o\{T(r, f)\}$ as $r \rightarrow \infty$ possibly outside a set of finite linear measure. We denote by $T(r)$ the maximum of $T(r, f)$ and $T(r, g)$. The notation $S(r)$ denotes any quantity satisfying $S(r)=o(T(r))$ as $r \longrightarrow \infty$, outside of a possible exceptional set of finite logarithmic measure.

A meromorphic function $a(z)$ is called a small function with respect to $f$, provided that $T(r, a)=S(r, f)$. The order and hyper order of meromorphic function $f$ are defined respectively by

$$
\sigma(f)=\limsup _{r \rightarrow \infty} \frac{\log T(r, f)}{\log r}
$$

and

$$
\sigma_{2}(f)=\limsup _{r \rightarrow \infty} \frac{\log \log T(r, f)}{\log r} .
$$

Let $f(z)$ and $g(z)$ be two non-constant meromorphic functions. Let $a(z)$ be a small function with respect to $f(z)$ and $g(z)$. We say that $f(z)$ and $g(z)$ share $a(z)$ CM (counting multiplicities) if $f(z)-a(z)$ and $g(z)-a(z)$ have the same zeros with the same multiplicities and we say that $f(z), g(z)$ share $a(z)$ IM (ignoring multiplicities) if we do not consider the multiplicities.

Received June 5, 2016, accepted September 12, 2017.

2010 Mathematics Subject Classification. Primary 30D35.

Key words and phrases. Meromorphic function, difference polynomial, uniqueness, weighted sharing. Corresponding author: Abhijit Banerjee. 
Define the difference of $f(z)$ by

$$
\Delta_{c} f(z)=f(z+c)-f(z)
$$

In 2010, Qi et al. [10] proved the following uniqueness theorem regarding shift operator.

Theorem A. Let $f$ and $g$ be transcendental entire functions of finite order, let c be a non-zero complex constant, and let $n \geq 6$ be an integer. If $f^{n}(z) f(z+c)$ and $g^{n}(z) g(z+c)$ share $z C M$, then $f(z) \equiv \operatorname{tg}(z)$ for a constant $t$ satisfying $t^{n+1}=1$.

In 2011 Zhang-Cao and Li [17] proved the difference counterparts of the above theorem in the following manner.

Theorem B. Let $f$ and $g$ be two non-constantentire functions offinite order, and let $n \geq 5$ be an integer. Suppose that $c$ is a non-zero complex constant such that $\Delta_{c} f(z) \not \equiv 0$ and $\Delta_{c} g(z) \not \equiv 0$. If $f^{n}(z) \Delta_{c} f(z)$ and $g^{n}(z) \Delta_{c} g(z)$ share $z C M$ and $g(z+c)$ and $g(z)$ share $0 C M$, then $f(z) \equiv \operatorname{tg}(z)$, where $t$ is a constant satisfying $t^{n+1}=1$.

Theorem C. Let $f$ and $g$ be non-constant entire functions of finite order, and let $n \geq 5$ be an integer. Suppose that $c$ is a non-zero complex constant such that $\Delta_{c} f(z) \not \equiv 0$ and $\Delta_{c} g(z) \not \equiv 0$. If $f^{n}(z) \Delta_{c} f(z)$ and $g^{n}(z) \Delta_{c} g(z)$ share 1 CM and $g(z+c)$ and $g(z)$ share 0 CM, then

(1) $f(z) \equiv \operatorname{tg}(z)$ for a constant $t$ satisfying $t^{n+1}=1$;

(2) $f(z)=c_{1} e^{a z}$ and $g(z)=c_{2} e^{-a z}$, where $a, c_{1}$ and $c_{2}$ are non-zero constants such that $\left(c_{1} c_{2}\right)^{n+1}\left(e^{a c}+e^{-a c}-2\right)=-1$.

In 2012 Liu-Liu and Cao [9] first considered the value distribution of the differentialdifference counterpart of the above theorems and obtained the following result.

Theorem D. Let $f(z)$ be a transcendental entire function of finite order, not a periodic function with period $c$ and $\alpha(z)$ be a small function with respect to $f(z)$. If $n \geq k+3$, then the differencedifferential polynomial $\left[f^{n}(z) \Delta_{c} f(z)\right]^{(k)}-\alpha(z)$ has infinitely many zeros.

In 2013, Wu [11] obtained the uniqueness result corresponding to Theorem $\mathrm{D}$ as follows.

Theorem E. Let $f(z)$ and $g(z)$ be transcendental entire functions of $\sigma_{2}(f)<1, n \geq 2 k+$ 7. Suppose that $c$ is a non-zero complex constant such that $\Delta_{c} f(z) \not \equiv 0$ and $\Delta_{c} g(z) \not \equiv 0$. If $\left[f^{n}(z) \Delta_{c} f(z)\right]^{(k)}$ and $\left[g^{n}(z) \Delta_{c} g(z)\right]^{(k)}$ share $1 C M$, then $f(z) \equiv \operatorname{tg}(z)$, where $t$ is a constant satisfying $t^{n+1}=1$. 
Theorem F. Let $f(z)$ and $g(z)$ be transcendental entire functions of $\sigma_{2}(f)<1, n \geq 5 k+$ 13. Suppose that $c$ is a non-zero complex constant such that $\Delta_{c} f(z) \not \equiv 0$ and $\Delta_{c} g(z) \not \equiv 0$. If $\left[f^{n}(z) \Delta_{c} f(z)\right]^{(k)}$ and $\left[g^{n}(z) \Delta_{c} g(z)\right]^{(k)}$ share 1 IM, then $f(z) \equiv \operatorname{tg}(z)$, where $t$ is a constant satisfying $t^{n+1}=1$.

We see that considering $k$-th derivative of the difference form in Theorem $\mathrm{C}$ the condition $g(z+c)$ and $g(z)$ share $0 \mathrm{CM}$ has been removed here by the author in [11], but we point out some gaps in the proof of Theorem 5 [11]. In p. 7, at the starting of the second stanza that is the line after equation (62), the author used $T\left(r, \frac{f}{g}\right)=T(r, f)+T(r, g)+O(1)$ and putting this expression in (62) the author derived the conclusion of the theorem. But we know that $T\left(r, \frac{f}{g}\right) \leq T(r, f)+T(r, g)+O(1)$. So it will be interesting to find the correct form of Theorems E-F. Here we have dealt with this problem. In the paper, in the corrected version of Theorems $\mathrm{E}$ and $\mathrm{F}$ we have reduced the lower bound of $n$. We diminish the lower bound in Theorem $\mathrm{D}$ as well. Finally combining Theorems C-D, we present a single theorem where sharing of a polynomial under much relaxed sharing hypothesis has been taken under consideration and thus we improve and unify Theorems C-D in a more compact form.

The relaxation has been done on the basis of the notion of weighted sharing obtained by I. Lahiri as follows.

Definition 1. [8] Let $k \in \mathbb{N} \cup\{0\} \cup\{\infty\}$. For $a \in \mathbb{C} \cup\{\infty\}$ we denote by $E_{k}(a ; f)$ the set of all $a$-points of $f$ where an $a$-point of multiplicity $m$ is counted $m$ times if $m \leq k$ and $k+1$ times if $m>k$. If $E_{k}(a ; f)=E_{k}(a ; g)$, we say that $f, g$ share the value $a$ with weight $k$.

The definition implies that if $f, g$ share a value $a$ with weight $k$, then $z_{0}$ is an $a$-point of $f$ with multiplicity $m(\leq k)$ if and only if it is an $a$-point of $g$ with multiplicity $m(\leq k)$ and $z_{0}$ is an a-point of $f$ with multiplicity $m(>k)$ if and only if it is an a-point of $g$ with multiplicity $n(>k)$, where $m$ is not necessarily equal to $n$.

We write $f, g$ share $(a, k)$ to mean that $f, g$ share the value $a$ with weight $k$. Clearly if $f$, $g$ share $(a, k)$ then $f, g$ share $(a, p)$ for any integer $p, 0 \leq p<k$. Also we note that $f, g$ share a value $a \mathrm{IM}$ or CM if and only if $f, g$ share $(a, 0)$ or $(a, \infty)$ respectively.

We now present the following theorems which are the main results of the paper.

Theorem 1. Let $f(z)$ be a transcendental entire function of finite order such that $\Delta_{c} f(z) \not \equiv 0$ and $\alpha(z)$ be a small function with respect to $f(z)$. If $n \geq k+2$, then the difference-differential polynomial $\left[f^{n}(z) \Delta_{c} f(z)\right]^{(k)}-\alpha(z)$ has infinitely many zeros.

Theorem 2. Let $f(z)$ and $g(z)$ be transcendental entire functions of finite order and $n, k$ be two positive integers. Suppose that $c$ is a non-zero complex constant such that $\Delta_{c} f(z) \not \equiv 0$ and $\Delta_{c} g(z) \not \equiv 0$. Let $\left[f^{n}(z) \Delta_{c} f(z)\right]^{(k)}$ and $\left[g^{n}(z) \Delta_{c} g(z)\right]^{(k)}$ share $\left(1, k_{1}\right)$ and one of the following conditions holds: 
(i) $k_{1} \geq 2$ and $n>2 k+5$;

(ii) $k_{1}=1$ and $n>\frac{5 k}{2}+6$;

(iii) $k_{1}=0$ and $n>5 k+11$.

Then one of the following two conclusions hold:

(1) $f^{n}(z) \Delta_{c} f(z) \equiv g^{n}(z) \Delta_{c} g(z)$;

(2) $f(z)=c_{1} e^{a z}$ and $g(z)=c_{2} e^{-a z}$, where $a, c_{1}$ and $c_{2}$ are non-zero constants such that $(-1)^{k}\left(c_{1} c_{2}\right)^{n+1}[(n+1) a]^{2 k}\left(2-e^{a c}-e^{-a c}\right)=1$.

Theorem 3. Let $f(z)$ and $g(z)$ be transcendental entire functions of finite order and $n$ be a positive integer such that $n \geq 5$. Suppose that $c$ is a non-zero complex constant such that $\Delta_{c} f(z) \not \equiv 0$ and $\Delta_{c} g(z) \not \equiv 0$. Let $f^{n}(z) \Delta_{c} f(z)-p(z)$ and $g^{n}(z) \Delta_{c} g(z)-p(z)$ share $(0,2)$, where $p(z)$ be a nonzero polynomial such that $\operatorname{deg}(p) \leq n-1$ and $g(z), g(z+c)$ share 0 CM. Now

(1) when $p(z)$ is a non-constant polynomial, then $f(z) \equiv \operatorname{tg}(z)$ for a constant $t$ satisfying $t^{n+1}=1$

(2) when $p(z)$ is a nonzero constant d, then one of the following two conclusions hold:

(i) $f(z) \equiv \operatorname{tg}(z)$ for a constant $t$ satisfying $t^{n+1}=1$;

(ii) $f(z)=c_{1} e^{a z}$ and $g(z)=c_{2} e^{-a z}$, where $a, c_{1}$ and $c_{2}$ are non-zero constants such that $\left(c_{1} c_{2}\right)^{n+1}\left(e^{a c}+e^{-a c}-2\right)=-d^{2}$.

We now explain following definitions and notations which are used in the paper.

Definition 2 ([5]). Let $a \in \mathbb{C} \cup\{\infty\}$. For $p \in \mathbb{N}$ we denote by $N(r, a ; f \mid \leq p)$ the counting function of those $a$-points of $f$ (counted with multiplicities) whose multiplicities are not greater than $p$. By $\bar{N}(r, a ; f \mid \leq p)$ we denote the corresponding reduced counting function.

In an analogous manner we can define $N(r, a ; f \mid \geq p)$ and $\bar{N}(r, a ; f \mid \geq p)$.

Definition 3 ([8]). Let $k \in \mathbb{N} \cup\{\infty\}$. We denote by $N_{k}(r, a ; f)$ the counting function of $a$-points of $f$, where an $a$-point of multiplicity $m$ is counted $m$ times if $m \leq k$ and $k$ times if $m>k$. Then $N_{k}(r, a ; f)=\bar{N}(r, a ; f)+\bar{N}(r, a ; f \mid \geq 2)+\cdots+\bar{N}(r, a ; f \mid \geq k)$. Clearly $N_{1}(r, a ; f)=\bar{N}(r, a ; f)$.

Definition 4. Let $a, b \in \mathbb{C} \cup\{\infty\}$. Let $p$ be a positive integer. We denote by $\bar{N}(r, a ; f|\geq p|$ $g=b)(\bar{N}(r, a ; f|\geq p| g \neq b))$ the reduced counting function of those $a$-points of $f$ with multiplicities $\geq p$, which are the $b$-points (not the $b$-points) of $g$.

Definition $5([6,8])$. Let $f$, $g$ share a value $a \mathrm{IM}$. We denote by $\bar{N}_{*}(r, a ; f, g)$ the reduced counting function of those $a$-points of $f$ whose multiplicities differ from the multiplicities of the corresponding $a$-points of $g$. 


\section{Lemmas}

Let $F, G$ be two non-constant meromorphic functions. Henceforth we shall denote by $H$ the following function

$$
H=\left(\frac{F^{\prime \prime}}{F^{\prime}}-\frac{2 F^{\prime}}{F-1}\right)-\left(\frac{G^{\prime \prime}}{G^{\prime}}-\frac{2 G^{\prime}}{G-1}\right) .
$$

Lemma 1 ([12]). Let $f(z)$ be a non-constant meromorphic function and let $a_{n}(z)(\not \equiv 0), a_{n-1}(z)$, $\ldots, a_{0}(z)$ be meromorphic functions such that $T\left(r, a_{i}(z)\right)=S(r, f)$ for $i=0,1,2, \ldots, n$. Then

$$
T\left(r, a_{n} f^{n}+a_{n-1} f^{n-1}+\ldots+a_{1} f+a_{0}\right)=n T(r, f)+S(r, f) .
$$

Lemma 2 ([13]). Let $f(z)$ and $g(z)$ be two non-constant meromorphic functions. Then

$$
N\left(r, \infty ; \frac{f}{g}\right)-N\left(r, \infty ; \frac{g}{f}\right)=N(r, \infty ; f)+N(r, 0 ; g)-N(r, \infty ; g)-N(r, 0 ; f) .
$$

Lemma 3 ([16]). Let $f(z)$ be a non-constant meromorphic function and $p, k$ be positive integers. Then

$$
\begin{aligned}
& N_{p}\left(r, 0 ; f^{(k)}\right) \leq T\left(r, f^{(k)}\right)-T(r, f)+N_{p+k}(r, 0 ; f)+S(r, f), \\
& N_{p}\left(r, 0 ; f^{(k)}\right) \leq k \bar{N}(r, \infty ; f)+N_{p+k}(r, 0 ; f)+S(r, f) .
\end{aligned}
$$

Lemma 4 ([7]). If $N\left(r, 0 ; f^{(k)} \mid f \neq 0\right)$ denotes the counting function of those zeros of $f^{(k)}(z)$ which are not the zeros of $f(z)$, where a zero of $f^{(k)}(z)$ is counted according to its multiplicity, then

$$
N\left(r, 0 ; f^{(k)} \mid f \neq 0\right) \leq k \bar{N}(r, \infty ; f)+N(r, 0 ; f \mid<k)+k \bar{N}(r, 0 ; f \mid \geq k)+S(r, f) .
$$

Lemma 5 ([3]). Let $f(z)$ be a meromorphic function of finite order $\sigma$, and let $c \in \mathbb{C} \backslash\{0\}$ be fixed. Then for each $\varepsilon>0$, we have

$$
m\left(r, \frac{f(z+c)}{f(z)}\right)+m\left(r, \frac{f(z)}{f(z+c)}\right)=O\left(r^{\sigma-1+\varepsilon}\right)=S(r, f) .
$$

The following lemma has little modifications of the original version (Theorem 2.1 of [3]) Lemma 6. Let $f(z)$ be a transcendental meromorphic function offinite order, $c \in \mathbb{C} \backslash\{0\}$ be fixed. Then

$$
T(r, f(z+c))=T(r, f)+S(r, f) .
$$

Lemma 7. Let $f(z), g(z)$ be two transcendental entire functions of finite order, $c \in \mathbb{C} \backslash\{0\}$ be finite complex constant such that $\Delta_{c} f(z) \not \equiv 0$ and $\Delta_{c} g(z) \not \equiv 0$. Let $n(\geq 1)$ be an integer such that $n>3$. If $g(z+c), g(z)$ share $0 C M$ and $f^{n}(z) \Delta_{c} f(z) \equiv g^{n}(z) \Delta_{c} g(z)$, then $f(z) \equiv \operatorname{tg}(z)$ for a constant $t$ with $t^{n+1}=1$. 
Proof. Proof of Lemma follows from the proof of Theorem 1.10 [17].

Lemma 8. Let $f(z)$ be a transcendental entire function of finite order, $c \in \mathbb{C} \backslash\{0\}$ be finite complex constants and $n \in \mathbb{N}$. Let $\Phi(z)=f^{n}(z) \Delta_{c} f(z)$, where $\Delta_{c} f(z) \not \equiv 0$. Then we have

$$
n T(r, f) \leq T(r, \Phi)-N\left(r, 0 ; \Delta_{c} f\right)+S(r, f) .
$$

Proof. Note that by Lemmas 2 and 5 we have

$$
\begin{aligned}
m\left(r, f^{n+1}\right) & =m\left(r, \frac{\Phi f}{\Delta_{c} f}\right) \\
& \leq m(r, \Phi)+m\left(r, \frac{f}{\Delta_{c} f}\right)+S(r, f) \\
& =m(r, \Phi)+T\left(r, \frac{f}{\Delta_{c} f}\right)-N\left(r, \infty ; \frac{f}{\Delta_{c} f}\right)+S(r, f) \\
& =m(r, \Phi)+T\left(r, \frac{\Delta_{c} f}{f}\right)-N\left(r, \infty ; \frac{f}{\Delta_{c} f}\right)+S(r, f) \\
& =m(r, \Phi)+N\left(r, \infty ; \frac{\Delta_{c} f}{f}\right)+m\left(r, \frac{\Delta_{c} f}{f}\right)-N\left(r, \infty ; \frac{f}{\Delta_{c} f}\right)+S(r, f) \\
& \leq m(r, \Phi)+N(r, 0 ; f)-N\left(r, 0 ; \Delta_{c} f\right)+S(r, f) \\
& \leq m(r, \Phi)+T(r, f)-N\left(r, 0 ; \Delta_{c} f\right)+S(r, f) .
\end{aligned}
$$

By Lemma 1 we get

$$
(n+1) T(r, f)=m\left(r, f^{n+1}\right) \leq T(r, \Phi)+T(r, f)-N\left(r, 0 ; \Delta_{c} f\right)+S(r, f),
$$

i.e.,

$$
n T(r, f) \leq T(r, \Phi)-N\left(r, 0 ; \Delta_{c} f\right)+S(r, f)
$$

This completes the Lemma.

Lemma 9. Let $f(z)$ be a transcendental entire function of finite order, $c \in \mathbb{C} \backslash\{0\}$ be finite complex constants and $n \in \mathbb{N}$ such that $n \geq 1$. Then $S\left(r, f^{n}(z) \Delta_{c} f(z)\right)=S(r, f(z))$, where $\Delta_{c} f(z) \not \equiv 0$.

Proof. By Lemmas 1 and 6 we have

$$
\begin{aligned}
T\left(r, f^{n}(z) \Delta_{c} f(z)\right) & \leq T\left(r, f^{n}(z)\right)+T\left(r, \Delta_{c} f(z)\right) \\
& \leq T\left(r, f^{n}(z)\right)+T(r, f(z+c))+T(r, f(z))+S(r, f(z)) \\
& \leq(n+2) T(r, f(z))+S(r, f(z)) .
\end{aligned}
$$

This shows that $T\left(r, f^{n}(z) \Delta_{c} f(z)\right)=O(T(r, f))$.

Also by Lemma 8 we have $T(r, f(z))=O\left(T\left(r, f^{n}(z) \Delta_{c} f(z)\right)\right)$. Thus we have

$$
S\left(r, f^{n}(z) \Delta_{c} f(z)\right)=S(r, f(z)) .
$$

This completes the proof. 
Lemma 10. Let $f(z), g(z)$ be two transcendental entire functions of finite order, $c \in \mathbb{C} \backslash\{0\}$ be finite complex constant such that $\Delta_{c} f(z) \not \equiv 0$ and $\Delta_{c} g(z) \not \equiv 0$ and let $n(\geq 1)$ and $k(\geq 1)$ be two integers such that $n>2 k+3$. Let $F(z)=\left[f^{n}(z) \Delta_{c} f(z)\right]^{(k)}$ and $G(z)=\left[g^{n}(z) \Delta_{c} g(z)\right]^{(k)}$. If $H \equiv 0$, then one of the following conclusions occur

(i) $\left[f^{n}(z) \Delta_{c} f(z)\right]^{(k)}\left[g^{n}(z) \Delta_{c} g(z)\right]^{(k)} \equiv 1$, where $\left[f^{n}(z) \Delta_{c} f(z)\right]^{(k)}$ and $\left[g^{n}(z) \Delta_{c} g(z)\right]^{(k)}$ share $1 C M$;

(ii) $f^{n}(z) \Delta_{c} f(z) \equiv g^{n}(z) \Delta_{c} g(z)$.

Proof. Since $H \equiv 0$, by integration we get

$$
\frac{1}{F-1} \equiv \frac{B G+A-B}{G-1},
$$

where $A, B$ are constants and $A \neq 0$. From (2.9) it is clear that $F$ and $G$ share $(1, \infty)$. We now consider following cases.

Case 1. Let $B \neq 0$ and $A \neq B$.

If $B=-1$, then from (2.9) we have

$$
F \equiv \frac{-A}{G-A-1}
$$

Therefore

$$
\bar{N}(r, A+1 ; G)=0 .
$$

So in view of Lemmas 3, 8 and the second fundamental theorem we get

$$
\begin{aligned}
n T(r, g) & \leq T\left(r, g^{n} \Delta_{c} g\right)-N\left(r, 0 ; \Delta_{c} g\right)+S(r, g) \\
& \leq T(r, G)+N_{k+1}\left(r, 0 ; g^{n} \Delta_{c} g\right)-\bar{N}(r, 0 ; G)-N\left(r, 0 ; \Delta_{c} g\right)+S(r, g) \\
& \leq \bar{N}(r, 0 ; G)+\bar{N}(r, A+1 ; G)+N_{k+1}\left(r, 0 ; g^{n} \Delta_{c} g\right)-\bar{N}(r, 0 ; G)-N\left(r, 0 ; \Delta_{c} g\right)+S(r, g) \\
& \leq N_{k+1}\left(r, 0 ; g^{n} \Delta_{c} g\right)-N\left(r, 0 ; \Delta_{c} g\right)+S(r, g) \\
& \leq N_{k+1}\left(r, 0 ; g^{n}\right)+N_{k+1}\left(r, 0 ; \Delta_{c} g\right)-N\left(r, 0 ; \Delta_{c} g\right)+S(r, g) \\
& \leq(k+1) \bar{N}(r, 0 ; g)+S(r, g) \\
& \leq(k+1) T(r, g)+S(r, g),
\end{aligned}
$$

which is a contradiction since $n>k+1$.

If $B \neq-1$, from (2.9) we obtain that

$$
F-\left(1+\frac{1}{B}\right) \equiv \frac{-A}{B^{2}\left[G+\frac{A-B}{B}\right]} .
$$

So

$$
\bar{N}\left(r, \frac{(B-A)}{B} ; G\right)=0 .
$$


Using Lemmas 3, 8 and the same argument as used in the case when $B=-1$ we can get a contradiction.

Case 2. Let $B \neq 0$ and $A=B$.

If $B=-1$, then from (2.9) we have

$$
F(z) G(z) \equiv 1
$$

i.e.,

$$
\left[f^{n}(z) \Delta_{c} f(z)\right]^{(k)}\left[g^{n}(z) \Delta_{c} g(z)\right]^{(k)} \equiv 1 .
$$

If $B \neq-1$, from (2.9) we have

$$
\frac{1}{F} \equiv \frac{B G}{(1+B) G-1}
$$

Therefore

$$
\bar{N}\left(r, \frac{1}{1+B} ; G\right)=\bar{N}(r, 0 ; F) .
$$

So in view of Lemmas 3, 5, 8 and the second fundamental theorem we get

$$
\begin{aligned}
n T(r, g) & \leq T\left(r, g^{n} \Delta_{c} g\right)-N\left(r, 0 ; \Delta_{c} g\right)+S(r, g) \\
& \leq T(r, G)+N_{k+1}\left(r, 0 ; g^{n} \Delta_{c} g\right)-\bar{N}(r, 0 ; G)-N\left(r, 0 ; \Delta_{c} g\right)+S(r, g) \\
& \leq \bar{N}(r, 0 ; G)+\bar{N}\left(r, \frac{1}{1+B} ; G\right)+N_{k+1}\left(r .0 ; g^{n} \Delta_{c} g\right)-\bar{N}(r, 0 ; G)-N\left(r, 0 ; \Delta_{c} g\right)+S(r, g) \\
& \leq(k+1) \bar{N}(r, 0 ; g)+\bar{N}(r, 0 ; F)+S(r, g) \\
& \leq(k+1) \bar{N}(r, 0 ; g)+(k+1) \bar{N}(r, 0 ; f)+N\left(r, 0 ; \Delta_{c} f\right)+S(r, f)+S(r, g) \\
& \leq(k+1) \bar{N}(r, 0 ; g)+(k+1) \bar{N}(r, 0 ; f)+T\left(r, \Delta_{c} f\right)+S(r, f)+S(r, g) \\
& \leq(k+1) \bar{N}(r, 0 ; g)+(k+1) \bar{N}(r, 0 ; f)+m\left(r, \Delta_{c} f\right)+S(r, f)+S(r, g) \\
& \leq(k+1) \bar{N}(r, 0 ; g)+(k+1) \bar{N}(r, 0 ; f)+m\left(r, \frac{\Delta_{c} f}{f}\right)+m(r, f)+S(r, f)+S(r, g) \\
& \leq(k+1) T(r, g)+(k+2) T(r, f)+S(r, f)+S(r, g) .
\end{aligned}
$$

Without loss of generality, we suppose that there exists a set $I$ with infinite measure such that $T(r, f) \leq T(r, g)$ for $r \in I$. So for $r \in I$ we have

$$
(n-2 k-3) T(r, g) \leq S(r, g)
$$

which is a contradiction since $n>2 k+3$.

Case 3. Let $B=0$. From (2.9) we obtain

$$
F \equiv \frac{G+A-1}{A} .
$$

If $A \neq 1$, then from (2.10) we obtain

$$
\bar{N}(r, 1-A ; G)=\bar{N}(r, 0 ; F) .
$$


We can similarly deduce a contradiction as in Case 2. Therefore $A=1$ and from (2.10) we obtain

$$
F(z) \equiv G(z)
$$

i.e.,

$$
\left[f^{n}(z) \Delta_{c} f(z)\right]^{(k)} \equiv\left[g^{n}(z) \Delta_{c} g(z)\right]^{(k)}
$$

From (2.6) we get

$$
f^{n}(z) \Delta_{c} f(z) \equiv g^{n}(z) \Delta_{c} g(z)+p_{1}(z),
$$

where $p_{1}(z)$ is a polynomial of degree at most $k-1$. Suppose $p_{1}(z) \not \equiv 0$. Then from (2.7) we have

$$
\frac{f^{n} \Delta_{c} f}{p_{1}} \equiv \frac{g^{n} \Delta_{c} g}{p_{1}}+1 .
$$

Now in view of Lemmas 5, 8 and the second fundamental theorem we have

$$
\begin{aligned}
n T(r, f) \leq & T\left(r, f^{n} \Delta_{c} f\right)-N\left(r, 0, \Delta_{c} f\right)+S(r, f) \\
\leq & T\left(r, \frac{f^{n} \Delta_{c} f}{p_{1}}\right)-N\left(r, 0, \Delta_{c} f\right)+S(r, f) \\
\leq & \bar{N}\left(r, 0 ; \frac{f^{n} \Delta_{c} f}{p_{1}}\right)+\bar{N}\left(r, \infty ; \frac{f^{n} \Delta_{c} f}{p_{1}}\right)+\bar{N}\left(r, 1 ; \frac{f^{n} \Delta_{c} f}{p_{1}}\right) \\
& -N\left(r, 0, \Delta_{c} f\right)+S(r, f) \\
\leq & \bar{N}(r, 0 ; f)+\bar{N}\left(r, 0 ; \Delta_{c} f\right)+\bar{N}\left(r, 0 ; \frac{g^{n} \Delta_{c} g}{p_{1}}\right)-N\left(r, 0, \Delta_{c} f\right) \\
& +S(r, f)+S(r, g) \\
\leq & \bar{N}(r, 0 ; f)+\bar{N}(r, 0 ; g)+\bar{N}\left(r, 0 ; \Delta_{c} g\right)+S(r, f)+S(r, g) \\
\leq & T(r, f)+T(r, g)+T\left(r, \Delta_{c} g\right)+S(r, f)+S(r, g) \\
= & T(r, f)+T(r, g)+m\left(r, \frac{\Delta_{c} g}{g}\right)+m(r, g)+S(r, f)+S(r, g) \\
\leq & T(r, f)+2 T(r, g)+S(r, f)+S(r, g) .
\end{aligned}
$$

Similarly we have

$$
n T(r, g) \leq 2 T(r, f)+T(r, g)+S(r, f)+S(r, g) .
$$

Therefore we get

$$
n[T(r, f)+T(r, g)] \leq 3[T(r, f)+T(r, g)]+S(r, f)+S(r, g),
$$

which is a contradiction since $n>3$. Hence $p_{1}(z) \equiv 0$ and so from (2.7) we obtain

$$
f^{n}(z) \Delta_{c} f(z) \equiv g^{n}(z) \Delta_{c} g(z) .
$$

This completes the proof. 
Lemma 11. Let $f(z), g(z)$ be two transcendental entire functions of finite order, $c \in \mathbb{C} \backslash\{0\}$ be finite complex constant such that $\Delta_{c} f(z) \not \equiv 0$ and $\Delta_{c} g(z) \not \equiv 0$ and let $n$ be an integer such that $n>3$. Let $F(z)=\frac{f^{n}(z) \Delta_{c} f(z)}{p(z)}$ and $G(z)=\frac{g^{n}(z) \Delta_{c} g(z)}{p(z)}$, where $p(z)$ is non-zero polynomial. If $g(z)$, $g(z+c)$ share $0 C M$ and $H \equiv 0$, then one of the following conclusions occur

(i) $f^{n}(z) \Delta_{c} f(z) g^{n}(z) \Delta_{c} g(z) \equiv p^{2}(z)$, where $f^{n}(z) \Delta_{c} f(z)-p(z)$ and $g^{n}(z) \Delta_{c} g(z)-p(z)$ share $0 \mathrm{CM}$;

(ii) $f(z) \equiv \operatorname{tg}(z)$ for a constant $t$ with $t^{n+1}=1$.

Proof. Since $H \equiv 0$, by integration we get

$$
\frac{1}{F-1} \equiv \frac{B G+A-B}{G-1}
$$

where $A, B$ are constants and $A \neq 0$. From (2.9) it is clear that $F$ and $G$ share $(1, \infty)$. We now consider following cases.

Case 1. Let $B \neq 0$ and $A \neq B$.

If $B=-1$, then from (2.9) we have

$$
F \equiv \frac{-A}{G-A-1}
$$

Therefore

$$
\bar{N}(r, A+1 ; G)=N(r, 0 ; p)=S(r, g) .
$$

So in view of Lemma 8 and the second fundamental theorem we get

$$
\begin{aligned}
n T(r, g) & \leq T\left(r, g^{n} \Delta_{c} g\right)-N\left(r, 0 ; \Delta_{c} g\right)+S(r, g) \\
& \leq T(r, G)-N\left(r, 0 ; \Delta_{c} g\right)+S(r, g) \\
& \leq \bar{N}(r, 0 ; G)+\bar{N}(r, A+1 ; G)-N\left(r, 0 ; \Delta_{c} g\right)+S(r, g) \\
& \leq \bar{N}(r, 0 ; g)+S(r, g) \leq T(r, g)+S(r, g),
\end{aligned}
$$

which is a contradiction since $n>1$.

If $B \neq-1$, from (2.9) we obtain that

$$
F-\left(1+\frac{1}{B}\right) \equiv \frac{-A}{B^{2}\left[G+\frac{A-B}{B}\right]} .
$$

So

$$
\bar{N}\left(r, \frac{(B-A)}{B} ; G\right)=S(r, g) .
$$

Using Lemma 8 and the same argument as used in the case when $B=-1$ we can get a contradiction. 
Case 2. Let $B \neq 0$ and $A=B$.

If $B=-1$, then from (2.9) we have

$$
F(z) G(z) \equiv 1,
$$

i.e.,

$$
f^{n}(z) \Delta_{c} f(z) g^{n}(z) \Delta_{c} g(z) \equiv p^{2}(z)
$$

where $f^{n}(z) \Delta_{c} f(z)-p(z)$ and $g^{n}(z) \Delta_{c} g(z)-p(z)$ share 0 CM.

If $B \neq-1$, from (2.9) we have

$$
\frac{1}{F} \equiv \frac{B G}{(1+B) G-1}
$$

Therefore

$$
\bar{N}\left(r, \frac{1}{1+B} ; G\right)=\bar{N}(r, 0 ; F)+S(r, f) .
$$

So in view of Lemmas 5, 8 and the second fundamental theorem we get

$$
\begin{aligned}
n T(r, g) & \leq T\left(r, g^{n} \Delta_{c} g\right)-N\left(r, 0 ; \Delta_{c} g\right)+S(r, g) \\
& \leq T(r, G)-N\left(r, 0 ; \Delta_{c} g\right)+S(r, g) \\
& \leq \bar{N}(r, 0 ; G)+\bar{N}\left(r, \frac{1}{1+B} ; G\right)-N\left(r, 0 ; \Delta_{c} g\right)+S(r, g) \\
& \leq \bar{N}(r, 0 ; g)+\bar{N}(r, 0 ; F)+S(r, g) \\
& \leq \bar{N}(r, 0 ; g)+\bar{N}(r, 0 ; f)+N\left(r, 0 ; \Delta_{c} f\right)+S(r, f)+S(r, g) \\
& \leq \bar{N}(r, 0 ; g)+\bar{N}(r, 0 ; f)+T\left(r, \Delta_{c} f\right)+S(r, f)+S(r, g) \\
& \leq T(r, g)+2 T(r, f)+S(r, f)+S(r, g) .
\end{aligned}
$$

So for $r \in I$ we have

$$
(n-3) T(r, g) \leq S(r, g) \text {, }
$$

which is a contradiction since $n>3$.

Case 3. Let $B=0$. From (2.9) we obtain

$$
F \equiv \frac{G+A-1}{A} .
$$

If $A \neq 1$, then from (2.10) we obtain

$$
\bar{N}(r, 1-A ; G)=\bar{N}(r, 0 ; F) .
$$

We can similarly deduce a contradiction as in Case 2. Therefore $A=1$ and from (2.10) we obtain

$$
F(z) \equiv G(z)
$$


i.e.,

$$
f^{n}(z) \Delta_{c} f(z) \equiv g^{n}(z) \Delta_{c} g(z) .
$$

Then by Lemma 7 we have $f(z) \equiv \operatorname{tg}(z)$ for a constant $t$ with $t^{n+1}=1$.

This completes the proof.

Lemma 12. Let $f(z), g(z)$ be two transcendental entire functions of finite order, $c \in \mathbb{C} \backslash\{0\}$ be finite complex constant such that $\Delta_{c} f(z) \not \equiv 0$ and $\Delta_{c} g(z) \not \equiv 0$. Let $n(\geq 1)$ and $k(\geq 1)$ be two integers such that $n>k$. If

$$
\left[f^{n}(z) \Delta_{c} f(z)\right]^{(k)}\left[g^{n}(z) \Delta_{c} g(z)\right]^{(k)} \equiv 1,
$$

then

$$
f(z)=c_{1} e^{a z}, \quad g(z)=c_{2} e^{-a z},
$$

where $a, c_{1}$ and $c_{2}$ are non-zero constants such that $(-1)^{k}\left(c_{1} c_{2}\right)^{n+1}[(n+1) a]^{2 k}\left(2-e^{a c}-e^{-a c}\right)=$ 1.

Proof. Suppose

$$
\left[f^{n}(z) \Delta_{c} f(z)\right]^{(k)}\left[g^{n}(z) \Delta_{c} g(z)\right]^{(k)} \equiv 1 .
$$

Since $n>k$, it follows that $f(z)$ and $g(z)$ have no zeros and so $f(z)$ and $g(z)$ take the form

$$
f(z)=e^{\alpha(z)} \text { and } g(z)=e^{\beta(z)},
$$

where $\alpha$ and $\beta$ are non-constant polynomials.

Let

$$
\alpha_{1}(z)=n \alpha(z)+\alpha(z+c)
$$

and

$$
\alpha_{2}(z)=\alpha(z+c)-\alpha(z)
$$

Then by induction we have

$$
\left[f^{n}(z) f(z+c)\right]^{(k)}=t_{1}\left(\alpha_{1}^{\prime}, \alpha_{1}^{\prime \prime}, \ldots, \alpha_{1}^{(k)}\right)(z) e^{\alpha_{1}(z)}
$$

and

$$
\left[f^{n+1}(z)\right]^{(k)}=t_{0}\left(\alpha^{\prime}, \alpha^{\prime \prime}, \ldots, \alpha^{(k)}\right)(z) e^{(n+1) \alpha(z)},
$$

where $t_{1}\left(\alpha_{1}^{\prime}, \alpha_{1}^{\prime \prime}, \ldots, \alpha_{1}^{(k)}\right)$ and $t_{0}\left(\alpha^{\prime}, \alpha^{\prime \prime}, \ldots, \alpha^{(k)}\right)$ are differential polynomials in $\alpha_{1}^{\prime}, \alpha_{1}^{\prime \prime}, \ldots, \alpha_{1}^{(k)}$ and $\alpha^{\prime}, \alpha^{\prime \prime}, \ldots, \alpha^{(k)}$ respectively. Obviously

$$
t_{1}\left(\alpha_{1}^{\prime}, \alpha_{1}^{\prime \prime}, \ldots, \alpha_{1}^{(k)}\right) \not \equiv 0
$$


and

$$
t_{0}\left(\alpha^{\prime}, \alpha^{\prime \prime}, \ldots, \alpha^{(k)}\right) \not \equiv 0
$$

and $\left[f^{n}(z) \Delta_{c} f(z)\right]^{(k)} \not \equiv 0$. Clearly $T\left(r, t_{i}\right)=S(r, f)$ for $i=0,1$ and

$$
\bar{N}\left(r, 0 ; t_{1}(z) e^{\alpha_{2}(z)}-t_{0}(z)\right)=0 .
$$

So from (2.13) and using second fundamental theorem for small functions (see [15]), we obtain

$$
T\left(r, e^{\alpha_{2}}\right) \leq \bar{N}\left(r, 0 ; e^{\alpha_{2}}\right)+\bar{N}\left(r, \infty ; e^{\alpha_{2}}\right)+\bar{N}\left(r, 0 ; t_{1} e^{\alpha_{2}}-t_{0}\right)+S(r, f)=S(r, f) .
$$

This shows that $\alpha_{2}(z)$ is a constant. Let $\alpha_{2}(z) \equiv C$, where $C \in \mathbb{C}$. Then $\alpha(z+c) \equiv \alpha(z)+C$ and so $\operatorname{deg}(\alpha)=1$. Similarly we can $\operatorname{prove}$ that $\operatorname{deg}(\beta)=1$. Assume now that

$$
f(z)=c_{1} e^{a z}, g(z)=c_{2} e^{b z},
$$

where $a, b, c_{1}$ and $c_{2}$ are non-zero constants. Applying (2.11) again we get $a=-b$ and

$$
(-1)^{k}\left(c_{1} c_{2}\right)^{n+1}[(n+1) a]^{2 k}\left(2-e^{a c}-e^{-a c}\right)=1 .
$$

Finally $f(z)$ and $g(z)$ take the form

$$
f(z)=c_{1} e^{a z}, g(z)=c_{2} e^{-a z},
$$

where $a, c_{1}$ and $c_{2}$ are non-zero constants such that $(-1)^{k}\left(c_{1} c_{2}\right)^{n+1}[(n+1) a]^{2 k}\left(2-e^{a c}-e^{-a c}\right)$ $=1$. This completes the proof.

Lemma 13. [14] Let $f_{j}(j=1,2,3)$ be a meromorphic and $f_{1}$ be non-constant. Suppose that

$$
\sum_{j=1}^{3} f_{j} \equiv 1
$$

and

$$
\sum_{j=1}^{3} N\left(r, 0 ; f_{j}\right)+2 \sum_{j=1}^{3} \bar{N}\left(r, \infty ; f_{j}\right)<(\lambda+o(1)) T(r),
$$

as $r \longrightarrow+\infty, r \in I, \lambda<1$ and $T(r)=\max _{1 \leq j \leq 3} T\left(r, f_{j}\right)$. Then $f_{2} \equiv 1$ or $f_{3} \equiv 1$.

Lemma 14. Let $f(z), g(z)$ be two transcendental entire functions of finite order, $c \in \mathbb{C} \backslash\{0\}$ be finite complex constant such that $\Delta_{c} f(z) \not \equiv 0$ and $\Delta_{c} g(z) \not \equiv 0$ and let $n(>1)$ be an integer. Let $p(z)$ be a non-zero polynomial with $\operatorname{deg}(p) \leq n-1, f^{n}(z) \Delta_{c} f(z)-p(z)$ and $g^{n}(z) \Delta_{c} g(z)-p(z)$ share $0 \mathrm{CM}$ and $\mathrm{g}(z+c), \mathrm{g}(z)$ share $0 \mathrm{CM}$. Now

(i) if $p(z)$ is not a constant, then $f^{n}(z) \Delta_{c} f(z) g^{n}(z) \Delta_{c} g(z) \not \equiv p^{2}(z)$, 
(ii) if $p(z)$ is a non-zero constant d and $f^{n}(z) \Delta_{c} f(z) g^{n}(z) \Delta_{c} g(z) \equiv p^{2}(z)$, then

$$
f(z)=c_{1} e^{a z}, \quad g(z)=c_{2} e^{-a z}
$$

where $a, c_{1}$ and $c_{2}$ are non-zero constants such that $\left(c_{1} c_{2}\right)^{n+1}\left(e^{a c}+e^{-a c}-2\right)=-d^{2}$.

Proof. Suppose

$$
f^{n}(z) \Delta_{c} f(z) g^{n}(z) \Delta_{c} g(z) \equiv p^{2}(z)
$$

We consider the following cases:

Case 1: $\operatorname{Let} \operatorname{deg}(p(z))=l(\geq 1)$.

Since $n \geq 2$, it follows that $N(r, 0 ; f)=O(\log r)$ and $N(r, 0 ; g)=O(\log r)$.

Let

$$
F_{1}(z)=\frac{f^{n}(z) \Delta_{c} f(z)}{p(z)} \text { and } G_{1}(z)=\frac{g^{n}(z) \Delta_{c} g(z)}{p(z)} .
$$

From (2.14) we get

$$
F_{1} G_{1} \equiv 1
$$

If $F_{1} \equiv c G_{1}$, where $c$ is a nonzero constant, then by (2.16), $F_{1}$ is a constant and so $f$ is a polynomial, which contradicts our assumption. Hence $F_{1} \not \equiv G_{1}$.

Let

$$
\Phi(z)=\frac{f^{n}(z) \Delta_{c} f(z)-p(z)}{g^{n}(z) \Delta_{c} g(z)-p(z)} .
$$

We deduce from (2.17) that

$$
\Phi \equiv e^{\beta^{*}}
$$

where $\beta^{*}$ is a polynomial.

Let $f_{1}=F_{1}, f_{2}=-e^{\beta^{*}} G_{1}$ and $f_{3}=e^{\beta^{*}}$. Here $f_{1}$ is transcendental. Now from (2.18), we have

$$
f_{1}+f_{2}+f_{3} \equiv 1
$$

Clearly $T\left(r, F_{1}\right)=T\left(r, G_{1}\right)+O(1)$ and so by Lemma 9 we have $S(r, f)=S(r, g)$. Also $T\left(r, f_{3}\right) \leq$ $2 T\left(r, F_{1}\right)+S(r, f)$ and so $S\left(r, f_{3}\right)$ can be replaced by $S(r, f)$. Since $g(z)$ and $g(z+c)$ share $0 \mathrm{CM}$, it follows that $N\left(r, \infty ; \frac{\Delta_{c} g}{g}\right)=0$.

Note that

$$
\begin{aligned}
N\left(r, 0 ; f_{1}\right)=N\left(r, 0 ; F_{1}\right) & \leq N\left(r, 0 ; \Delta_{c} f(z)\right)+O(\log r) \\
& \leq T\left(r, \Delta_{c} f\right)+S(r, f)=T(r, f)+S(r, f)
\end{aligned}
$$

and

$$
N\left(r, 0 ; f_{2}\right)=N\left(r, 0 ; G_{1}\right)=N\left(r, 0 ; g^{n+1} \frac{\Delta_{c} g}{g}\right) \leq N\left(r, 0 ; \frac{\Delta_{c} g}{g}\right)+O(\log r)
$$




$$
\leq T\left(r, \frac{\Delta_{c} g}{g}\right)+S(r, g)=m\left(r, \frac{\Delta_{c} g}{g}\right)+S(r, g)=S(r, g) .
$$

Hence by Lemma 8 we get

$$
\begin{aligned}
\sum_{j=1}^{3} N\left(r, 0 ; f_{j}\right)+2 \sum_{j=1}^{3} \bar{N}\left(r, \infty ; f_{j}\right) & \leq N\left(r, 0 ; F_{1}\right)+N\left(r, 0 ; e^{\beta^{*}} G_{1}\right)+O(\log r) \\
& \leq T(r, f)+S(r, f) \leq \frac{1}{n} T\left(r, f_{1}\right)+S\left(r, f_{1}\right) \\
& \leq(\lambda+o(1)) T(r),
\end{aligned}
$$

as $r \longrightarrow+\infty, r \in I, \lambda=\frac{1}{n}<1$ and $T(r)=\max _{1 \leq j \leq 3} T\left(r, f_{j}\right)$.

So by Lemma 13, we get either $e^{\beta^{*}} G_{1} \equiv-1$ or $e^{\beta^{*}} \equiv 1$. But here the only possibility is that $e^{\beta^{*}} G_{1} \equiv-1$, i.e., $g^{n}(z) \Delta_{c} g(z) \equiv-e^{-\beta^{*}(z)} p(z)$ and so from (2.14) we obtain

$$
F_{1} \equiv e^{\gamma_{1}^{*}} G_{1}
$$

i.e.,

$$
f^{n}(z) \Delta_{c} f(z) \equiv e^{\gamma_{1}^{*}(z)} g^{n}(z) \Delta_{c} g(z)
$$

where $\gamma_{1}^{*}$ is a non-constant polynomial. Now from (2.14) we get

$$
f^{n}(z) \Delta_{c} f(z) \equiv c e^{\frac{1}{2} \gamma_{1}^{*}(z)} p(z), \quad g^{n}(z) \Delta_{c} g(z) \equiv c e^{-\frac{1}{2} \gamma_{1}^{*}(z)} p(z),
$$

where $c= \pm 1$.

Since $N(r, 0 ; f)=O(\log r)$ and $N(r, 0 ; g)=O(\log r)$, so we can take

$$
f(z)=h_{1}(z) e^{\alpha_{1}(z)}, \quad g(z)=h_{2}(z) e^{\beta_{1}(z)},
$$

where $h_{1}$ and $h_{2}$ are non-zero polynomials and $\alpha_{1}, \beta_{1}$ are two non-constant polynomials.

Since $\operatorname{deg}(p) \leq n-1$, from (2.19) and (2.20) we conclude that both $h_{1}$ and $h_{2}$ are non-zero constants.

So we can rewrite $f$ and $g$ as follows:

$$
f(z)=e^{\alpha(z)}, \quad g(z)=e^{\beta(z)},
$$

where $\alpha$ and $\beta$ are non-constant polynomials.

Now from (2.14) we have

$$
\left(e^{\alpha(z+c)-\alpha(z)}-1\right)\left(e^{\beta(z+c)-\beta(z)}-1\right) \equiv p^{2}(z) e^{-(n+1)[\alpha(z)+\beta(z)]} .
$$

Note that

$$
N\left(r, 1 ; e^{\alpha(z+c)-\alpha(z)}\right)=O(\log r) \text { and } N\left(r, 1 ; e^{\beta(z+c)-\beta(z)}\right)=O(\log r) .
$$


Let

$$
\phi(z)=e^{\alpha(z+c)-\alpha(z)} \text { and } \psi(z)=e^{\beta(z+c)-\beta(z)} .
$$

Clearly either both $\phi(z)$ and $\psi(z)$ are constants or both are transcendental entire functions. Suppose $\phi(z) \not \equiv$ constant and $\psi(z) \not \equiv$ constant. Now by second fundamental theorem we have

$$
\begin{aligned}
T(r, \phi) & \leq \bar{N}(r, 0 ; \phi)+\bar{N}(r, \infty ; \phi)+\bar{N}(r, 1 ; \phi)+S(r, \phi) \\
& \leq O(\log r)+S(r, \phi)=S(r, \phi),
\end{aligned}
$$

which is a contradiction. Therefore $\phi(z) \equiv$ constant. Similarly we can prove that $\psi(z) \equiv$ constant. Hence $p^{2}(z) e^{-(n+1)[\alpha(z)+\beta(z)]} \equiv$ constant, which is impossible.

Case 2: Let $p(z)$ be a non-zero constant $d$.

In this case we see that $f(z)$ and $g(z)$ have no zeros and so we can take $f(z)$ and $g(z)$ as follows:

$$
f(z)=e^{\alpha(z)}, \quad g(z)=e^{\beta(z)},
$$

where $\alpha$ and $\beta$ are non-constant polynomials.

Now from (2.14) we get

$$
\left(e^{\alpha(z+c)-\alpha(z)}-1\right)\left(e^{\beta(z+c)-\beta(z)}-1\right) \equiv d^{2} e^{-(n+1)[\alpha(z)+\beta(z)]} .
$$

We conclude from (2.23) that $e^{\alpha(z+c)-\alpha(z)}-1$ has no zeros. Let $\phi(z)=e^{\alpha(z+c)-\alpha(z)}$. Then $\phi(z) \neq$ $0,1, \infty$ for any $z \in \mathbb{C}$. By Picard's theorem, $\phi$ is a constant and so $\operatorname{deg}(\alpha)=1$. Similarly we can prove that $\operatorname{deg}(\beta)=1$. Assume now that

$$
f(z)=c_{1} e^{a z}, g(z)=c_{2} e^{b z},
$$

where $a, b, c_{1}$ and $c_{2}$ are non-zero constants. Applying (2.14) again we get $a=-b$ and

$$
\left(c_{1} c_{2}\right)^{n+1}\left(e^{a c}+e^{-a c}-2\right)=-d^{2} .
$$

Finally $f(z)$ and $g(z)$ take the form

$$
f(z)=c_{1} e^{a z}, g(z)=c_{2} e^{-a z},
$$

where $a, c_{1}$ and $c_{2}$ are non-zero constants such that $\left(c_{1} c_{2}\right)^{n+1}\left(e^{a c}+e^{-a c}-2\right)=-d^{2}$. This completes the proof.

Lemma 15 ([1]). If $f$, g be two non-constant meromorphic functions such that they share $(1,1)$. Then

$$
2 \bar{N}_{L}(r, 1 ; f)+2 \bar{N}_{L}(r, 1 ; g)+\bar{N}_{E}^{(2}(r, 1 ; f)-\bar{N}_{f>2}(r, 1 ; g) \leq N(r, 1 ; g)-\bar{N}(r, 1 ; g) .
$$


Lemma $16([2])$. Let $f$, $g$ share $(1,1)$. Then

$$
\bar{N}_{f>2}(r, 1 ; g) \leq \frac{1}{2} \bar{N}(r, 0 ; f)+\frac{1}{2} \bar{N}(r, \infty ; f)-\frac{1}{2} N_{0}\left(r, 0 ; f^{\prime}\right)+S(r, f),
$$

where $N_{0}\left(r, 0 ; f^{\prime}\right)$ is the counting function of those zeros of $f^{\prime}$ which are not the zeros of $f(f-1)$.

Lemma 17 ([2]). Let $f$ and $g$ be two non-constant meromorphic functions sharing $(1,0)$. Then

$$
\bar{N}_{L}(r, 1 ; f)+2 \bar{N}_{L}(r, 1 ; g)+\bar{N}_{E}^{(2}(r, 1 ; f)-\bar{N}_{f>1}(r, 1 ; g)-\bar{N}_{g>1}(r, 1 ; f) \leq N(r, 1 ; g)-\bar{N}(r, 1 ; g) .
$$

Lemma 18 ([2]). Let $f$, $g$ share $(1,0)$. Then

$$
\bar{N}_{L}(r, 1 ; f) \leq \bar{N}(r, 0 ; f)+\bar{N}(r, \infty ; f)+S(r, f)
$$

Lemma 19 ([2]). Let $f$, $g$ share $(1,0)$. Then

(i) $\bar{N}_{f>1}(r, 1 ; g) \leq \bar{N}(r, 0 ; f)+\bar{N}(r, \infty ; f)-N_{0}\left(r, 0 ; f^{\prime}\right)+S(r, f)$

(ii) $\bar{N}_{g>1}(r, 1 ; f) \leq \bar{N}(r, 0 ; g)+\bar{N}(r, \infty ; g)-N_{0}\left(r, 0 ; g^{\prime}\right)+S(r, g)$.

\section{Proofs of the Theorems}

Proof of Theorem 1. Let $F(z)=\left[f^{n}(z) \Delta_{c} f(z)\right]^{(k)}$. In view of Lemmas 3, 8 and by the second theorem for small functions (see [15]) we get

$$
\begin{aligned}
n T(r, f) \leq & T\left(r, f^{n} \Delta_{c} f\right)-N\left(r, 0 ; \Delta_{c} f\right)+S(r, f) \\
\leq & T(r, F)+N_{k+1}\left(r, 0 ; f^{n} \Delta_{c} f\right)-\bar{N}(r, 0 ; F)-N\left(r, 0 ; \Delta_{c} f\right)+S(r, f) \\
\leq & \bar{N}(r, 0 ; F)+\bar{N}(r, \alpha(z) ; F)+N_{k+1}\left(r, 0 ; f^{n} \Delta_{c} f\right)-\bar{N}(r, 0 ; F)-N\left(r, 0 ; \Delta_{c} f\right) \\
& +(\varepsilon+o(1)) T(r, f) \\
\leq & (k+1) \bar{N}(r, 0 ; f)+N\left(r, 0 ; \Delta_{c} f\right)+\bar{N}(r, \alpha(z) ; F)-N\left(r, 0 ; \Delta_{c} f\right) \\
& +(\varepsilon+o(1)) T(r, f) \\
\leq & (k+1) T(r, f)+\bar{N}(r, \alpha(z) ; F)+(\varepsilon+o(1)) T(r, f),
\end{aligned}
$$

for all $\varepsilon>0$. Take $\varepsilon<1$. Since $n \geq k+2$, from above one can easily say that $F(z)-\alpha(z)$ has infinitely many zeros. This completes the proof.

Proof of Theorem 2. Let $F(z)=\left[f^{n}(z) \Delta_{c} f(z)\right]^{(k)}$ and $G(z)=\left[g^{n}(z) \Delta_{c} g(z)\right]^{(k)}$. It follows that $F$ and $G$ share $\left(1, k_{1}\right)$. 
Case 1. Let $H \not \equiv 0$.

Subcase 1.1. $k_{1} \geq 1$.

From (2.1) it can be easily calculated that the possible poles of $H$ occur at (i) multiple zeros of $F$ and $G$, (ii) those 1 points of $F$ and $G$ whose multiplicities are different, (iii) zeros of $F^{\prime}\left(G^{\prime}\right)$ which are not the zeros of $F(F-1)(G(G-1))$.

Since $H$ has only simple poles we get

$$
\begin{aligned}
N(r, \infty ; H) \leq & \bar{N}_{*}(r, 1 ; F, G)+\bar{N}(r, 0 ; F \mid \geq 2)+\bar{N}(r, 0 ; G \mid \geq 2) \\
& +\bar{N}_{0}\left(r, 0 ; F^{\prime}\right)+\bar{N}_{0}\left(r, 0 ; G^{\prime}\right),
\end{aligned}
$$

where $\bar{N}_{0}\left(r, 0 ; F^{\prime}\right)$ is the reduced counting function of those zeros of $F^{\prime}$ which are not the zeros of $F(F-1)$ and $\bar{N}_{0}\left(r, 0 ; G^{\prime}\right)$ is similarly defined.

Let $z_{0}$ be a simple zero of $F-1$. Then $z_{0}$ is a simple zero of $G-1$ and a zero of $H$. So

$$
N(r, 1 ; F \mid=1) \leq N(r, 0 ; H) \leq N(r, \infty ; H)+S(r, f)+S(r, g) .
$$

While $k_{1} \geq 2$, using (3.3) and (3.4) we get

$$
\begin{aligned}
\bar{N}(r, 1 ; F) \leq & N(r, 1 ; F \mid=1)+\bar{N}(r, 1 ; F \mid \geq 2) \\
\leq & \bar{N}(r, 0 ; F \mid \geq 2)+\bar{N}(r, 0 ; G \mid \geq 2)+\bar{N}_{*}(r, 1 ; F, G) \\
& +\bar{N}(r, 1 ; F \mid \geq 2)+\bar{N}_{0}\left(r, 0 ; F^{\prime}\right)+\bar{N}_{0}\left(r, 0 ; G^{\prime}\right)+S(r, f)+S(r, g) .
\end{aligned}
$$

Now in view of Lemma 4 we get

$$
\begin{aligned}
& \bar{N}_{0}\left(r, 0 ; G^{\prime}\right)+\bar{N}(r, 1 ; F \mid \geq 2)+\bar{N}_{*}(r, 1 ; F, G) \\
& \quad \leq \bar{N}_{0}\left(r, 0 ; G^{\prime}\right)+\bar{N}(r, 1 ; F \mid \geq 2)+\bar{N}(r, 1 ; F \mid \geq 3) \\
& \quad=\bar{N}_{0}\left(r, 0 ; G^{\prime}\right)+\bar{N}(r, 1 ; G \mid \geq 2)+\bar{N}(r, 1 ; G \mid \geq 3) \\
& \quad \leq \bar{N}_{0}\left(r, 0 ; G^{\prime}\right)+N(r, 1 ; G)-\bar{N}(r, 1 ; G) \\
& \quad \leq N\left(r, 0 ; G^{\prime} \mid G \neq 0\right) \leq \bar{N}(r, 0 ; G)+S(r, g),
\end{aligned}
$$

Hence using (3.5), (3.22), Lemmas 3, 5 and 8 we get from second fundamental theorem that

$$
\begin{aligned}
n T(r, f) \leq & T\left(r, f^{n} \Delta_{c} f\right)-N\left(r, 0 ; \Delta_{c} f\right)+S(r, f) \\
\leq & T(r, F)+N_{k+2}\left(r, 0 ; f^{n} \Delta_{c} f\right)-N_{2}(r, 0 ; F)-N\left(r, 0 ; \Delta_{c} f\right)+S(r, f) \\
\leq & \bar{N}(r, 0 ; F)+\bar{N}(r, 1 ; F)+N_{k+2}\left(r, 0 ; f^{n} \Delta_{c} f\right)-N\left(r, 0 ; \Delta_{c} f\right) \\
& -N_{2}(r, 0 ; F)-N_{0}\left(r, 0 ; F^{\prime}\right)+S(r, f) \\
\leq & \bar{N}(r, 0 ; F)+N_{k+2}\left(r, 0 ; f^{n} \Delta_{c} f\right)+\bar{N}(r, 0 ; F \mid \geq 2)+\bar{N}(r, 0 ; G \mid \geq 2)+\bar{N}(r, 1 ; F \mid \geq 2) \\
& +\bar{N}_{*}(r, 1 ; F, G)+\bar{N}_{0}\left(r, 0 ; G^{\prime}\right)-N\left(r, 0 ; \Delta_{c} f\right)-N_{2}(r, 0 ; F)+S(r, f)+S(r, g)
\end{aligned}
$$




$$
\begin{aligned}
\leq & N_{k+2}\left(r, 0 ; f^{n} \Delta_{c} f\right)+N_{2}(r, 0 ; G)-N\left(r, 0 ; \Delta_{c} f\right)+S(r, f)+S(r, g) \\
\leq & N_{k+2}\left(r, 0 ; f^{n} \Delta_{c} f\right)+N_{k+2}\left(r, 0 ; g^{n} \Delta_{c} g\right)-N\left(r, 0 ; \Delta_{c} f\right)+S(r, f)+S(r, g) \\
\leq & (k+2) \bar{N}(r, 0 ; f)+N\left(r, 0 ; \Delta_{c} f\right)+(k+2) \bar{N}(r, 0 ; g)+N\left(r, 0 ; \Delta_{c} g\right) \\
& -N\left(r, 0 ; \Delta_{c} f\right)+S(r, f)+S(r, g) \\
\leq & (k+2) T(r, f)+(k+2) T(r, g)+T\left(r, \Delta_{c} g\right)+S(r, f)+S(r, g) \\
\leq & (k+2) T(r, f)+(k+2) T(r, g)+m\left(r, \Delta_{c} g\right)+S(r, f)+S(r, g) \\
\leq & (k+2) T(r, f)+(k+2) T(r, g)+m\left(r, \frac{\Delta_{c} g}{g}\right)+m(r, g)+S(r, f)+S(r, g) \\
\leq & (k+2) T(r, f)+(k+3) T(r, g)+S(r, f)+S(r, g) \\
\leq & (2 k+5) T(r)+S(r) .
\end{aligned}
$$

In a similar way we can obtain

$$
n T(r, g) \leq(2 k+5) T(r)+S(r) \text {. }
$$

Combining (3.7) and (3.8) we see that

$$
n T(r) \leq(2 k+5) T(r)+S(r)
$$

i.e.,

$$
(n-2 k-5) T(r) \leq S(r) .
$$

Since $n>2 k+5$, (3.9) leads to a contradiction.

While $k_{1}=1$, using Lemmas 4, 15, 16, (3.3) and (3.4) we get

$$
\begin{aligned}
\bar{N}(r, 1 ; F) \leq & N(r, 1 ; F \mid=1)+\bar{N}_{L}(r, 1 ; F)+\bar{N}_{L}(r, 1 ; G)+\bar{N}_{E}^{(2}(r, 1 ; F) \\
\leq & \bar{N}(r, 0 ; F \mid \geq 2)+\bar{N}(r, 0 ; G \mid \geq 2)+\bar{N}_{*}(r, 1 ; F, G)+\bar{N}_{L}(r, 1 ; F)+\bar{N}_{L}(r, 1 ; G) \\
& +\bar{N}_{E}^{(2}(r, 1 ; F)+\bar{N}_{0}\left(r, 0 ; F^{\prime}\right)+\bar{N}_{0}\left(r, 0 ; G^{\prime}\right)+S(r, f)+S(r, g) \\
\leq & \bar{N}(r, 0 ; F \mid \geq 2)+\bar{N}(r, 0 ; G \mid \geq 2)+2 \bar{N}_{L}(r, 1 ; F)+2 \bar{N}_{L}(r, 1 ; G)+\bar{N}_{E}^{(2}(r, 1 ; F) \\
& +\bar{N}_{0}\left(r, 0 ; F^{\prime}\right)+\bar{N}_{0}\left(r, 0 ; G^{\prime}\right)+S(r, f)+S(r, g) \\
\leq & \bar{N}(r, 0 ; F \mid \geq 2)+\bar{N}(r, 0 ; G \mid \geq 2)+\bar{N}_{F>2}(r, 1 ; G)+N(r, 1 ; G)-\bar{N}(r, 1 ; G) \\
& +\bar{N}_{0}\left(r, 0 ; F^{\prime}\right)+\bar{N}_{0}\left(r, 0 ; G^{\prime}\right)+S(r, f)+S(r, g) \\
\leq & \bar{N}(r, 0 ; F \mid \geq 2)+\frac{1}{2} \bar{N}(r, 0 ; F)+\bar{N}(r, 0 ; G \mid \geq 2)+N(r, 1 ; G)-\bar{N}(r, 1 ; G) \\
& +\bar{N}_{0}\left(r, 0 ; G^{\prime}\right)+\bar{N}_{0}\left(r, 0 ; F^{\prime}\right)+S(r, f)+S(r, g) \\
\leq & \bar{N}(r, 0 ; F \mid \geq 2)+\frac{1}{2} \bar{N}(r, 0 ; F)+\bar{N}(r, 0 ; G \mid \geq 2)+N\left(r, 0 ; G^{\prime} \mid G \neq 0\right)+\bar{N}_{0}\left(r, 0 ; F^{\prime}\right) \\
& +S(r, f)+S(r, g) \\
\leq & \bar{N}(r, 0 ; F \mid \geq 2)+\frac{1}{2} \bar{N}(r, 0 ; F)+N_{2}(r, 0 ; G)+\bar{N}_{0}\left(r, 0 ; F^{\prime}\right)+S(r, f)+S(r, g) . \quad(3
\end{aligned}
$$


Hence using (3.10), Lemmas 3, 5 and 8 we get from second fundamental theorem that

$$
\begin{aligned}
n T(r, f) \leq & T\left(r, f^{n} \Delta_{c} f\right)-N\left(r, 0 ; \Delta_{c} f\right)+S(r, f) \\
\leq & T(r, F)+N_{k+2}\left(r, 0 ; f^{n} \Delta_{c} f\right)-N_{2}(r, 0 ; F)-N\left(r, 0 ; \Delta_{c} f\right)+S(r, f) \\
\leq & \bar{N}(r, 0 ; F)+\bar{N}(r, 1 ; F)+N_{k+2}\left(r, 0 ; f^{n} \Delta_{c} f\right)-N_{2}(r, 0 ; F)-N_{0}\left(r, 0 ; F^{\prime}\right) \\
& -N\left(r, 0 ; \Delta_{c} f\right)+S(r, f) \\
\leq & N_{2}(r, 0 ; F)+\frac{1}{2} \bar{N}(r, 0 ; F)+N_{k+2}\left(r, 0 ; f^{n} \Delta_{c} f\right)+N_{2}(r, 0 ; G)-N_{2}(r, 0 ; F) \\
& -N\left(r, 0 ; \Delta_{c} f\right)+S(r, f)+S(r, g) \\
\leq & N_{k+2}\left(r, 0 ; f^{n} \Delta_{c} f\right)+\frac{1}{2} \bar{N}(r, 0 ; F)+N_{2}(r, 0 ; G)-N\left(r, 0 ; \Delta_{c} f\right)+S(r, f)+S(r, g) \\
\leq & N_{k+2}\left(r, 0 ; f^{n} \Delta_{c} f\right)+N_{k+2}\left(r, 0 ; g^{n} \Delta_{c} g\right)+\frac{1}{2} N_{k+1}\left(r, 0 ; f^{n} \Delta_{c}\right) \\
& -N\left(r, 0 ; \Delta_{c} f\right)+S(r, f)+S(r, g) \\
\leq & \frac{3 k+5}{2} \bar{N}(r, 0 ; f)+\frac{1}{2} N\left(r, 0 ; \Delta_{c} f\right)+(k+2) \bar{N}(r, 0 ; g)+N\left(r, 0 ; \Delta_{c} g\right)+S(r, f)+S(r, g) \\
\leq & \frac{3 k+6}{2} T(r, f)+(k+3) T(r, g)+S(r, f)+S(r, g) \\
\leq & \left(\frac{5 k}{2}+6\right) T(r)+S(r) .
\end{aligned}
$$

In a similar way we can obtain

$$
n T(r, g) \leq\left(\frac{5 k}{2}+6\right) T(r)+S(r) .
$$

Combining (3.11) and (3.12) we see that

$$
\left(n-\frac{5 k}{2}-6\right) T(r) \leq S(r)
$$

Since $n>\frac{5 k}{2}+6$, (3.13) leads to a contradiction.

Subcase 1.2. $k_{1}=0$. Here (3.4) changes to

$$
N_{E}^{1)}(r, 1 ; F \mid=1) \leq N(r, 0 ; H) \leq N(r, \infty ; H)+S(r, F)+S(r, G) .
$$

Using Lemmas 4, 17, 18, 19, (3.3) and (3.14) we get

$$
\begin{aligned}
\bar{N}(r, 1 ; F) \leq & N_{E}^{1)}(r, 1 ; F)+\bar{N}_{L}(r, 1 ; F)+\bar{N}_{L}(r, 1 ; G)+\bar{N}_{E}^{(2}(r, 1 ; F) \\
\leq & \bar{N}(r, 0 ; F \mid \geq 2)+\bar{N}(r, 0 ; G \mid \geq 2)+\bar{N}_{*}(r, 1 ; F, G)+\bar{N}_{L}(r, 1 ; F)+\bar{N}_{L}(r, 1 ; G) \\
& +\bar{N}_{E}^{(2}(r, 1 ; F)+\bar{N}_{0}\left(r, 0 ; F^{\prime}\right)+\bar{N}_{0}\left(r, 0 ; G^{\prime}\right)+S(r, f)+S(r, g) \\
\leq & \bar{N}(r, 0 ; F \mid \geq 2)+\bar{N}(r, 0 ; G \mid \geq 2)+2 \bar{N}_{L}(r, 1 ; F)+2 \bar{N}_{L}(r, 1 ; G)+\bar{N}_{E}^{(2}(r, 1 ; F) \\
& +\bar{N}_{0}\left(r, 0 ; F^{\prime}\right)+\bar{N}_{0}\left(r, 0 ; G^{\prime}\right)+S(r, f)+S(r, g)
\end{aligned}
$$




$$
\begin{aligned}
\leq & \bar{N}(r, 0 ; F \mid \geq 2)+\bar{N}(r, 0 ; G \mid \geq 2)+\bar{N}_{F>1}(r, 1 ; G)+\bar{N}_{G>1}(r, 1 ; F)+\bar{N}_{L}(r, 1 ; F) \\
& +N(r, 1 ; G)-\bar{N}(r, 1 ; G)+\bar{N}_{0}\left(r, 0 ; F^{\prime}\right)+\bar{N}_{0}\left(r, 0 ; G^{\prime}\right)+S(r, f)+S(r, g) \\
\leq & N_{2}(r, 0 ; F)+\bar{N}(r, 0 ; F)+N_{2}(r, 0 ; G)+N(r, 1 ; G)-\bar{N}(r, 1 ; G) \\
& +\bar{N}_{0}\left(r, 0 ; G^{\prime}\right)+\bar{N}_{0}\left(r, 0 ; F^{\prime}\right)+S(r, f)+S(r, g) \\
\leq & N_{2}(r, 0 ; F)+\bar{N}(r, 0 ; F)+N_{2}(r, 0 ; G)+N\left(r, 0 ; G^{\prime} \mid G \neq 0\right)+\bar{N}_{0}\left(r, 0 ; F^{\prime}\right) \\
& +S(r, f)+S(r, g) \\
\leq & N_{2}(r, 0 ; F)+\bar{N}(r, 0 ; F)+N_{2}(r, 0 ; G)+\bar{N}(r, 0 ; G)+\bar{N}_{0}\left(r, 0 ; F^{\prime}\right) \\
& +S(r, f)+S(r, g) .
\end{aligned}
$$

Hence using (3.15), Lemmas 3, 5 and 8 we get from second fundamental theorem that

$$
\begin{aligned}
n T(r, f) \leq & T\left(r, f^{n} \Delta_{c} f\right)-N\left(r, 0 ; \Delta_{c} f\right)+S(r, f) \\
\leq & T(r, F)+N_{k+2}\left(r, 0 ; f^{n} \Delta_{c} f\right)-N_{2}(r, 0 ; F)+S(r, f) \\
\leq & \bar{N}(r, 0 ; F)+\bar{N}(r, 1 ; F)+N_{k+2}\left(r, 0 ; f^{n} \Delta_{c} f\right)-N_{2}(r, 0 ; F)-N_{0}\left(r, 0 ; F^{\prime}\right) \\
& -N\left(r, 0 ; \Delta_{c} f\right)+S(r, f) \\
\leq & N_{2}(r, 0 ; F)+2 \bar{N}(r, 0 ; F)+N_{k+2}\left(r, 0 ; f^{n} \Delta_{c} f\right) \\
& +N_{2}(r, 0 ; G)+\bar{N}(r, 0 ; G)-N_{2}(r, 0 ; F)-N\left(r, 0 ; \Delta_{c} f\right)+S(r, f)+S(r, g) \\
\leq & N_{k+2}\left(r, 0 ; f^{n} \Delta_{c} f\right)+2 \bar{N}(r, 0 ; F)+N_{2}(r, 0 ; G) \\
& +\bar{N}(r, 0 ; G)-N\left(r, 0 ; \Delta_{c} f\right)+S(r, f)+S(r, g) \\
\leq & N_{k+2}\left(r, 0 ; f^{n} \Delta_{c} f\right)+2 N_{k+1}\left(r, 0 ; f^{n} \Delta_{c} f\right)+N_{k+2}\left(r, 0 ; g^{n} \Delta_{c} g\right)+N_{k+1}\left(r, 0 ; g^{n} \Delta_{c} g\right) \\
& -N\left(r, 0 ; \Delta_{c} f\right)+S(r, f)+S(r, g) \\
\leq & (3 k+4) \bar{N}(r, 0 ; f)+2 N\left(r, 0 ; \Delta_{c} f\right)+(2 k+3) \bar{N}(r, 0 ; g)+2 N\left(r, 0 ; \Delta_{c} g\right)+S(r, f)+S(r, g) \\
\leq & (3 k+6) T(r, f)+(2 k+5) T(r, g)+S(r, f)+S(r, g) \\
\leq & (5 k+11) T(r)+S(r) .
\end{aligned}
$$

In a similar way we can obtain

$$
n T(r, g) \leq(5 k+11) T(r)+S(r) .
$$

Combining (3.16) and (3.17) we see that

$$
(n-5 k-11) T(r) \leq S(r) .
$$

Since $n>5 k+11$, (3.18) leads to a contradiction.

Case 2. Let $H \equiv 0$. Then the theorem follows from Lemmas 10 and 12 . This completes the proof. 
Proof of Theorem 3. Let $F(z)=\frac{f^{n}(z) \Delta_{c} f(z)}{p(z)}$ and $G(z)=\frac{g^{n}(z) \Delta_{c} g(z)}{p(z)}$. It follows that $F$ and $G$ share $(1,2)$ except for the zeros of $p(z)$.

Case 1. Let $H \not \equiv 0$.

From (2.1) we obtain

$$
N(r, \infty ; H) \leq \bar{N}_{*}(r, 1 ; F, G)+\bar{N}(r, 0 ; F \mid \geq 2)+\bar{N}(r, 0 ; G \mid \geq 2)+\bar{N}_{0}\left(r, 0 ; F^{\prime}\right)+\bar{N}_{0}\left(r, 0 ; G^{\prime}\right) .
$$

Let $z_{0}$ be a simple zero of $F-1$ such that $p\left(z_{0}\right) \neq 0$. Then $z_{0}$ is a simple zero of $G-1$ and a zero of $H$. So

$$
N(r, 1 ; F \mid=1) \leq N(r, 0 ; H) \leq N(r, \infty ; H)+S(r, f)+S(r, g) .
$$

Using (3.19) and (3.20) we get

$$
\begin{aligned}
\bar{N}(r, 1 ; F) \leq & \bar{N}(r, 0 ; F \mid \geq 2)+\bar{N}(r, 0 ; G \mid \geq 2)+\bar{N}_{*}(r, 1 ; F, G) \\
& +\bar{N}(r, 1 ; F \mid \geq 2)+\bar{N}_{0}\left(r, 0 ; F^{\prime}\right)+\bar{N}_{0}\left(r, 0 ; G^{\prime}\right)+S(r, f)+S(r, g) .
\end{aligned}
$$

Now in view of Lemma 4 we get

$$
\begin{aligned}
\bar{N}_{0}\left(r, 0 ; G^{\prime}\right)+\bar{N}(r, 1 ; F \mid \geq 2)+\bar{N}_{*}(r, 1 ; F, G) & \leq \bar{N}_{0}\left(r, 0 ; G^{\prime}\right)+\bar{N}(r, 1 ; F \mid \geq 2)+\bar{N}(r, 1 ; F \mid \geq 3) \\
& \leq N\left(r, 0 ; G^{\prime} \mid G \neq 0\right) \leq \bar{N}(r, 0 ; G)+S(r, g) .
\end{aligned}
$$

Note that since $g(z)$ and $g(z+c)$ share $0 \mathrm{CM}$, it follows that $N\left(r, \infty ; \frac{\Delta_{c} g}{g}\right)=0$.

Hence using (3.5), (3.22), Lemmas 5 and 8 we get from second fundamental theorem that

$$
\begin{aligned}
n T(r, f) & \leq T(r, F)-N\left(r, 0 ; \Delta_{c} f\right)+S(r, f) \\
& \leq \bar{N}(r, 0 ; F)+\bar{N}(r, 1 ; F)-N_{0}\left(r, 0 ; F^{\prime}\right)-N\left(r, 0 ; \Delta_{c} f\right)+S(r, f) \\
& \leq N_{2}(r, 0 ; F)+N_{2}(r, 0 ; G)-N\left(r, 0 ; \Delta_{c} f\right)+S(r, f)+S(r, g) \\
& \leq N_{2}\left(r, 0 ; f^{n} \Delta_{c} f\right)+N_{2}\left(r, 0 ; g^{n} \Delta_{c} g\right)-N\left(r, 0 ; \Delta_{c} f\right)+S(r, f)+S(r, g) \\
& \leq 2 \bar{N}(r, 0 ; f)+N\left(r, 0 ; \Delta_{c} f\right)+N_{2}\left(r, 0 ; g^{n+1} \frac{\Delta_{c} g}{g}\right)-N\left(r, 0 ; \Delta_{c} f\right)+S(r, f)+S(r, g) \\
& \leq 2 \bar{N}(r, 0 ; f)+N_{2}\left(r, 0 ; g^{n+1}\right)+N_{2}\left(r, 0 ; \frac{\Delta_{c} g}{g}\right)+S(r, f)+S(r, g) \\
& \leq 2 T(r, f)+2 T(r, g)+T\left(r, \frac{\Delta_{c} g}{g}\right)+S(r, f)+S(r, g) \\
& \leq 2 T(r, f)+2 T(r, g)+m\left(r, \frac{\Delta_{c} g}{g}\right)+S(r, f)+S(r, g) \\
& \leq 2 T(r, f)+2 T(r, g)+S(r, f)+S(r, g) .
\end{aligned}
$$

By Lemma 1

$$
(n+1) T(r, g)=T\left(r, g^{n+1}\right)=m\left(r, g^{n+1}\right) \leq m\left(r, \frac{g^{n+1}}{G}\right)+m(r, G)
$$




$$
\begin{aligned}
& \leq m\left(r, \frac{g}{\Delta_{c} g}\right)+T(r, G)+O(\log r) \\
& \leq T\left(r, \frac{\Delta_{c} g}{g}\right)+T(r, G)+S(r, g) \\
& =m\left(r, \frac{\Delta_{c} g}{g}\right)+T(r, G)+S(r, g)=T(r, G)+S(r, g) .
\end{aligned}
$$

In a similar way we can obtain

$$
\begin{aligned}
(n & +1) T(r, g) \leq T(r, G)+S(r, g) \\
& \leq \bar{N}(r, 0 ; G)+\bar{N}(r, 1 ; G)-N_{0}\left(r, 0 ; G^{\prime}\right)+S(r, f) \\
& \leq N_{2}(r, 0 ; G)+N_{2}(r, 0 ; F)+S(r, f)+S(r, g) \\
& \leq N_{2}\left(r, 0 ; g^{n+1} \frac{\Delta_{c} g}{g}\right)+N_{2}\left(r, 0 ; f^{n} \Delta_{c} f\right)+S(r, f)+S(r, g) \\
& \leq 2 \bar{N}(r, 0 ; g)+2 \bar{N}(r, 0 ; f)+N\left(r, 0 ; \Delta_{c} f\right)+S(r, f)+S(r, g) \\
& \leq 2 T(r, f)+2 T(r, g)+T\left(r, \Delta_{c} f\right)+S(r, f)+S(r, g) \\
& \leq 2 T(r, f)+2 T(r, g)+m\left(r, \frac{\Delta_{c} f}{f}\right)+m(r, f)+S(r, f)+S(r, g) \\
& \leq 3 T(r, f)+2 T(r, g)+S(r, f)+S(r, g) .
\end{aligned}
$$

Combining (3.23) and (3.24) we see that

$$
(n-5) T(r, f)+(n-4) T(r, g) \leq S(r, f)+S(r, g) .
$$

Since $n \geq 5$, (3.25) leads to a contradiction.

Case 2. Let $H \equiv 0$. Then the theorem follows from Lemmas 11, 7 and 14 . This completes the proof.

\section{Acknowledgement}

This research work is supported by the Council Of Scientific and Industrial Research, Extramural Research Division, CSIR Complex, Pusa, New Delhi-110012, India, under the sanction project no. 25 (0229)/14/EMR-II.

\section{References}

[1] T. C. Alzahary and H. X. Yi, Weighted value sharing and a question of I. Lahiri, Complex Var. Theory Appl., 49 (15) (2004), 1063-1078.

[2] A. Banerjee, Meromorphic functions sharing one value, Int. J. Math. Math. Sci., 22 (2005), 3587-3598.

[3] Y. M. Chiang and S. J. Feng, On the Nevanlinna characteristic $f(z+\eta)$ and difference equations in complex plane, Ramanujan J., 16 (2008), 105-129.

[4] W. K. Hayman, Meromorphic Functions, The Clarendon Press, Oxford (1964). 
[5] I. Lahiri, Value distribution of certain differential polynomials, Int. J. Math. Math. Sci., 28 (2001), 83-91.

[6] I. Lahiri, Weighted sharing and uniqueness of meromorphic functions, Nagoya Math. J., 161 (2001), $193-206$.

[7] I. Lahiri and S. Dewan, Value distribution of the product of a meromorphic function and its derivative, Kodai Math. J., 26 (2003), 95-100.

[8] I. Lahiri, Weighted value sharing and uniqueness of meromorphic functions, Complex Var. Theory Appl., 46 (2001), 241-253.

[9] K. Liu, X. L. Liu and T. B. Cao, Some results on zeros and uniqueness of difference-differential polynomials, Appl. Math. J. Chinese Univ., 27 (1) (2012), 94-104.

[10] X. G. Qi, L. Z. Yang and K. Liu, Uniqueness and periodicity of meromorphic functions concerning the difference operator, Com. Math. Appl., 60 (6) (2010), 1739-1746.

[11] C. Wu, Uniqueness of entire functions concerning difference operator, Abstract and Applied Analysis, Vol. 2013, Art. Id 240369, 9 pages.

[12] C. C. Yang, On deficiencies of differential polynomials II, Math. Z., 125 (1972), 107-112.

[13] L. Yang, Value Distribution Theory, Springer-Verlag and Science Press, 1993.

[14] C. C. Yang and H. X. Yi, Uniqueness Theory of Meromorphic Functions, Kluwer Academic Publishers, Dordrecht/Boston/London, 2003.

[15] K. Yamanoi, The second main theorem for small functions and related problems, Acta Math., 192 (2004), 225-294.

[16] Q. C. Zhang, Meromorphic function that shares one small function with its derivative, J. Inequal. Pure Appl. Math., 6 (4) (2005), Art.116 [ ONLINE http://jipam.vu.edu.au/].

[17] J. L. Zhang, Z. S. Gao and S. Li, Distribution of zeros and shares values of difference operator, Annal. Polon. Math., 102 (3) (2011), 213-221.

Department of Mathematics, University of Kalyani, West Bengal 741235, India.

E-mail: abanerjee_kal@yahoo.co.in; abanerjee_kal@rediffmail.com

Department of Mathematics, Raiganj University, Raiganj, West Bengal-733134, India.

E-mail:sm05math@gmail.com; sujoy.katwa@gmail.com; smajumder05@yahoo.in 